\title{
One-Pot Three-Component Coupling Reaction of $\alpha$-Amino Aryl Ketones, Indoles, and Perbromomethane Under Mild Conditions
}

\author{
De Chen ${ }^{\dagger}$, Hao Lu${ }^{\dagger}$, Yuxuan Liu ${ }^{\dagger}$,Wei Deng ${ }^{*}$, Renhua Qiu* and Jiannan Xiang * \\ College of Chemistry and Chemical Engineering, Hunan University, Changsha, China
}

A simple and efficient one-pot three-component cascade reaction of $\alpha$-amino aryl ketones, indoles, and $\mathrm{CBr}_{4}$ in moderate to good yields has been developed. This new strategy exhibits excellent mild reaction conditions and step-economy, easily accessible reactants, and simultaneous construction of three different new bonds $(\mathrm{C}=\mathrm{N}, \mathrm{C}-\mathrm{C}$, and $\mathrm{N}-\mathrm{Br})$ in a single step. It is worth noting that the protocol developed provides a simple and practical tool for the construction of diverse indole-containing heterocyclic frameworks, indicating its potential applications in medicinal and material chemistry.

Keywords: one-pot three-component, a-amino aryl ketones, indoles and perbromomethane, step-economy, C-H bond functionalization

\section{INTRODUCTION}

As one of the most important heterocycles, indole is widely present in natural products and medicines due to its remarkable biological activity, such as antibacterial (Van Order and Lindwall., 1942; Bell et al., 1994), anti-obesity (Sashidhara et al., 2012), antimicrobial (Sivaprasad et al., 2006), vaginal spermicide (Paira et al., 2009), and apoptosis in acute myelogenous leukemia (AML) (Contractor et al., 2005). The functionalization of the indole core mainly occurs at the N1, C2, and C3 positions (Bandini and Eichholzer, 2009; Joucla and Djakovitch., 2009; Bartoli et al., 2010; Dalpozzo., 2015; Sandtorv., 2015; Deka et al., 2020). Among them, the $C 3$ position modification of indoles is mainly achieved by transition metal-catalyzed C-H bond functionalization (Phipps et al., 2008; Leitch et al., 2017; Ye et al., 2020). In recent years, transition metal-catalyzed C-H functionalization at the $\mathrm{C} 3$ position of indoles has become a field of extensive research, and tremendous progress has been made in this regard (Kumar et al., 2021).

From the perspective of simplicity, the oxidative cross-dehydrogenation coupling reaction has become a very good tool for constructing complex molecules through simple reaction materials (Li., 2009; Scheuermann., 2010; Yeung and Dong., 2011; Girard et al., 2014; Song et al., 2017; Wang et al., 2021). Easy-to-prepare and cheap $\alpha$-amino carbonyl units are widely present in many natural products and drug molecules (Ohfune., 1992). However, there are a few reports as the starting material of oxidative cross-dehydrogenation coupling reactions. In 2012, Li group developed a C-H oxidative/ cross-coupling strategy of $\alpha$-amino carbonyls with indoles to selectively obtain 2 -(1H-indol-3-yl)-2imino-carbonyls under the $\mathrm{Cu}(\mathrm{I}) / \mathrm{TBHP}$ catalytic system (Wu et al., 2012; Scheme 1A). In the same year, the Li group continued to use the visible light photoredox strategy to realize the $\mathrm{C}-\mathrm{H}$ functionalization of $\alpha$-amino aryl ketones with indoles under $\mathrm{Ru}(\mathrm{bpy})_{3} \mathrm{Cl}_{2}$ catalysis and obtained 2-(1H-indol-3-yl)-2-amino-carbonyl compounds (Wang et al., 2012; Scheme 1B). After that, Feng group chose a cheaper $\mathrm{Fe}(\mathrm{III})$ catalyst and also realized C-H functionalization of $\alpha$-amino aryl ketones 


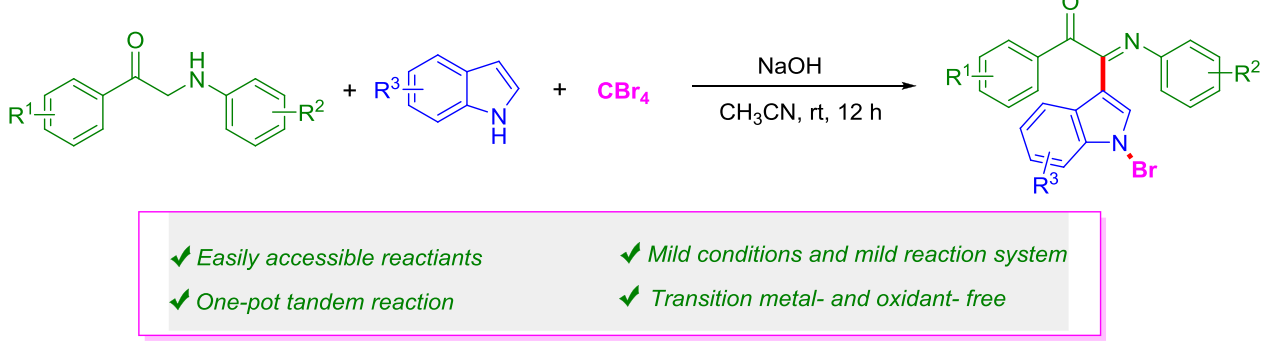

GRAPHICAL ABSTRACT |

Previous work (C-H functionalization reactions by adding transition metal and oxidant)

A $\mathrm{Cu}(\mathrm{I}) / \mathrm{TBHP}$-catalyzed $\mathrm{C}-\mathrm{H}$ oxidative/cross-coupling.

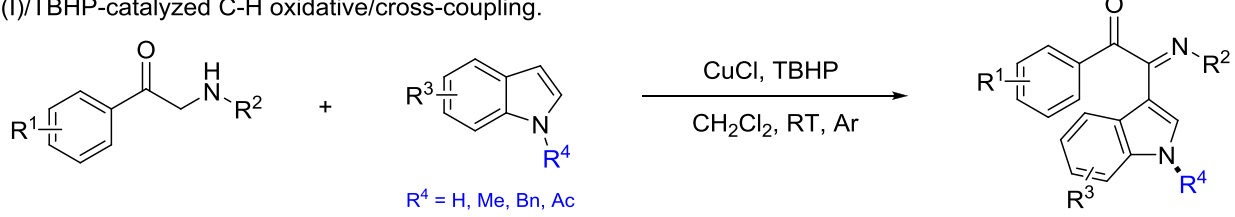

B $\mathrm{Ru}(\mathrm{II}) / \mathrm{O}_{2}$-catalyzed $\mathrm{C}-\mathrm{H}$ oxidative/cross-coupling.<smiles>[R][R]1C=Cc2ccn([Z20])c2C=C1</smiles>

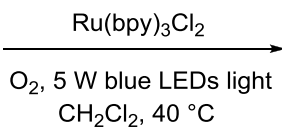

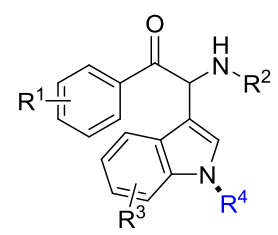

C $\mathrm{Fe}(\mathrm{III}) / \mathrm{TBHP}$-catalyzed $\mathrm{C}-\mathrm{H}$ oxidative/cross-coupling.<smiles>[R][R]1ccc2ccn([R])c2c1</smiles>
$\underset{\mathrm{CH}_{3} \mathrm{CN}, \mathrm{RT}, \text { Air }}{\stackrel{\mathrm{Fe}\left(\mathrm{ClO}_{4}\right)_{3}, \mathrm{TBHP}}{\longrightarrow}}$

$$
\mathrm{R}^{4}=\mathrm{H}, \mathrm{Me}, \mathrm{Bn}
$$<smiles>[R]NC(C(=O)c1cc[R1]([R])cc1)C1=CN([Z2])[R]([R])c2ccccc21</smiles>

D This work (Transition-metal-free, one-pot three-component system)
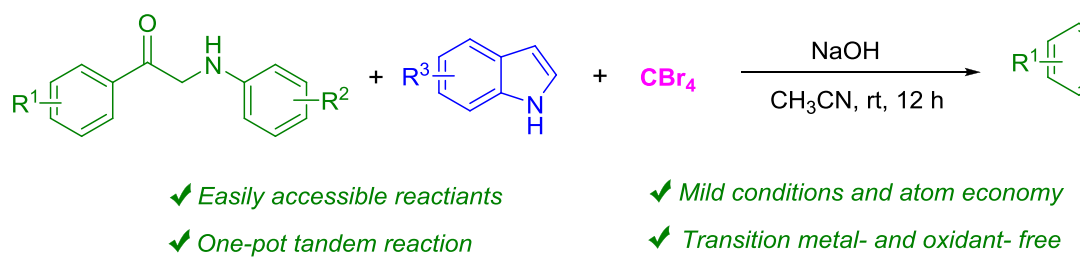

SCHEME 1 | C-H bond functionalization of a-amino aryl ketones and indoles.

with indoles in 2016 (Zhang et al., 2016; Scheme 1C). However, these synthetic methods all use transition metals and additional oxidants, so it is necessary to explore more green ways. This field aims to develop more efficient, green, and practical $\mathrm{C}-\mathrm{H}$ functionalization methods and expand its application range. Very recently, our group has also repeatedly reported the application of indoles in organic synthesis, such as direct synthesis of 3,3-diaryl benzofuranones (Tang et al., 2019), $\mathrm{N}$-aryl-1-amino indoles (Ou et al., 2021), and 3,3'- diindolylmethanes (DIMs) (Yang et al., 2020) by using indoles as the starting material.

Herein, we report a more effective and green method for the transition metal-free $\mathrm{C}-\mathrm{H}$ bond functionalization reaction of $\alpha$ amino aryl ketones, indoles, and $\mathrm{CBr}_{4}$ under mild conditions (Scheme 1D). This new methodology of green chemistry has several advantages, such as transition metal-free, cheap, and environmental benign reagents, mild reaction conditions, and step-economy. 
TABLE 1 | Optimization of the reaction conditions. ${ }^{\text {a }}$
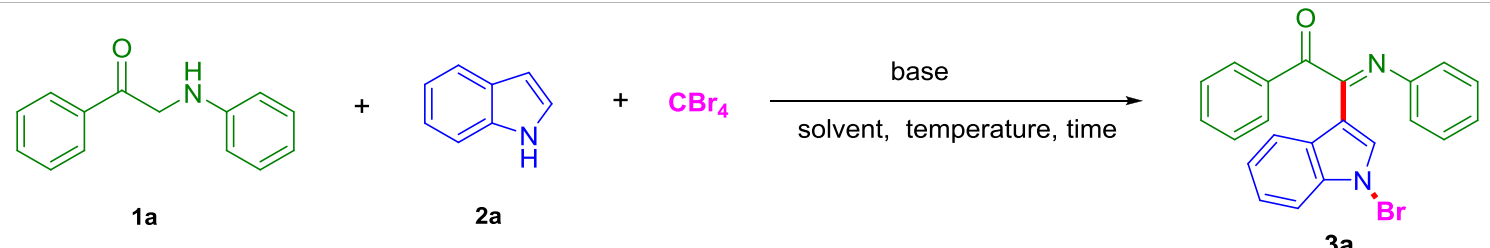

3a

\begin{tabular}{|c|c|c|c|c|}
\hline Entry & Solvent & Temperature $\left({ }^{\circ} \mathrm{C}\right)$ & Base & Yield $(\%)^{b}$ \\
\hline 1 & DCM & 40 & $\mathrm{NaOH}$ & 37 \\
\hline 2 & $\mathrm{PhMe}$ & 40 & $\mathrm{NaOH}$ & 21 \\
\hline 3 & DMF & 40 & $\mathrm{NaOH}$ & Trace \\
\hline 5 & DCE & 40 & $\mathrm{NaOH}$ & 39 \\
\hline 6 & DMSO & 40 & $\mathrm{NaOH}$ & Trace \\
\hline 7 & THF & 40 & $\mathrm{NaOH}$ & Trace \\
\hline 10 & $\mathrm{Et}_{2} \mathrm{O}$ & 40 & $\mathrm{NaOH}$ & Trace \\
\hline 11 & $\mathrm{MeOH}$ & 40 & $\mathrm{NaOH}$ & N.R \\
\hline 12 & $\mathrm{EtOH}$ & 40 & $\mathrm{NaOH}$ & N.R \\
\hline 13 & $\mathrm{MeCN}$ & RT & $\mathrm{NaOH}$ & 68 \\
\hline 14 & $\mathrm{MeCN}$ & 50 & $\mathrm{NaOH}$ & 27 \\
\hline 15 & $\mathrm{MeCN}$ & RT & LDA & N.R \\
\hline
\end{tabular}

${ }^{a}$ Reaction condition: All reactions were carried out with 1-phenyl-2-(phenylamino)ethan-1-one (1a) (0.3 mmol), $1 \mathrm{H}$-indole (2a) (0.3 mmol), $C B r_{4}(0.6 \mathrm{mmol})$, and base (1.2 mmol) in solvent $(2 \mathrm{ml})$ at certain temperature for $12 \mathrm{~h}$.

${ }^{b}$ /solated yield.

${ }^{c}$ Reaction for $6 \mathrm{~h}$.

${ }^{d}$ Reaction for $18 h$

\section{RESULTS AND DISCUSSION}

Initially, the reaction of 1-phenyl-2-(phenylamino)ethan-1-one (1a), $\mathrm{CBr}_{4}$ with $1 \mathrm{H}$-indole (2a) was selected as a model reaction to optimize the reaction conditions. The different bases, temperatures, times, and solvents were attempted to synthesize (E)-2-(1-bromo-1H-indol-3-yl)-1-phenyl-2-(phenylimino)ethan1-one (3a). The results are listed in Table 1. It was found that the reaction readily proceeded in $\mathrm{CH}_{2} \mathrm{Cl}_{2}$ using $\mathrm{NaOH}$ as a base leading to produce (E)-2-(1-bromo-1H-indol-3-yl)-1-phenyl-2(phenylimino)ethan-1-one (3a) in 37\% yield (Table 1, entry 1). This result prompted us to further search for the optimal reaction conditions. Then, we investigated the organic solvents, such as PhMe, DMF, MeCN, DCE, DMSO, THF, 1,4-dioxane, CYH (cyclohexane), $\mathrm{Et}_{2} \mathrm{O}, \mathrm{MeOH}$ and $\mathrm{EtOH}$ (Table 1, entries 2-12). The most effective solvent was $\mathrm{MeCN}$, which could give $3 \mathrm{a}$ in $61 \%$ yield (entry 4). Furthermore, temperature also affected the reaction. It was found that room temperature was an appropriate temperature for the reaction, and $3 \mathrm{a}$ was obtained in $68 \%$ yield (Table 1, entry 13). Higher temperatures did not significantly improve the yield (Table 1, entry 14). Lithium diisopropylamide (LDA) replaced $\mathrm{NaOH}$ as the dehydrogenation medium, and it was found that it did not participate in the reaction (Table 1, entry 15). In order to improve $3 \mathrm{a}$ yield, we have also checked the reaction using two additional bases, $\mathrm{LiOH}$ and $\mathrm{KOH}$. However, the low yield of 3a $(37 \%, 41 \%)$ was obtained, respectively (Table 1 , entries 16-17). Reaction time played an important role in the reaction. However, in this reaction, no matter whether prolonged or shortened the reaction time, the yield of $3 \mathrm{a}$ could not be improved (Table 1, entries 18-19).

On the basis of the optimized reaction conditions, the synthesis of various 2-(1-bromo- $1 H$-indol-3-yl)-2-iminocarbonyls was examined by the reactions of $a$-amino aryl ketones and $\mathrm{CBr}_{4}$ with $1 \mathrm{H}$-indole (2a) in $\mathrm{MeCN}$ at room temperature by using $\mathrm{NaOH}$ as a base. The results are listed in Table 2. It was found that $\alpha$-amino aryl ketones containing electron-donating groups, such as $\mathrm{Me}$ and $\mathrm{OMe}$, on the ortho- or para-positions of aromatic rings afforded the corresponding products in moderate to good yields (3b-3d). The $\alpha$-amino aryl ketones containing electron-withdrawing groups, such as $\mathrm{Cl}$, Br, I, and $\mathrm{CF}_{3}$, on the ortho-, meta-, or para-positions of the phenyl group could also give satisfactory yields (3e-3L). Naphthyl $\alpha$-amino aryl ketones can also produce corresponding products in moderate yields (Table $2,3 \mathrm{~m}$ ). The aromatic amines containing electron-donating groups or electron-withdrawing groups, such as $\mathrm{Me}$ and $\mathrm{Cl}$, on the metaor para-positions could also give satisfactory yield $(3 n-3 p)$. The $5-\mathrm{Me}$ and $6-\mathrm{Cl}$ of substituted indoles were selected as indole substitution groups to be tested. The corresponding products yields of $3 \mathrm{q}-3 \mathrm{r}$ were found to be good. After comparison, we 
TABLE 2 | Synthesis of 2-(1-bromo-1H-indol-3-yl)-2-imino-carbonyls from $\alpha$-amino aryl ketones., a,b

$$
\text { (1) }
$$<smiles>O=C(/C(=N/c1ccccc1)c1cn(Br)c2ccccc12)c1ccccc1</smiles>

$3 a, 68 \%$<smiles>O=C(/C(=N/c1ccccc1)c1cn(Br)c2ccccc12)c1ccc(F)cc1</smiles>

$3 e, 58 \%$<smiles>O=C(/C(=N/c1ccccc1)c1cn(Br)c2ccccc12)c1cccc(Br)c1</smiles>

3i, $65 \%$<smiles>O=C(/C(=N/c1ccccc1)c1cn(Br)c2ccccc12)c1ccc2ccccc2c1</smiles>

$3 m, 47 \%$<smiles>Cc1ccc2c(c1)c(/C(=N\c1ccccc1)C(=O)c1ccccc1)cn2Br</smiles>

$3 q, 78 \%$<smiles>Cc1ccccc1C(=O)/C(=N/c1ccccc1)c1cn(Br)c2ccccc12</smiles>

3b, $86 \%$<smiles>O=C(/C(=N/c1ccccc1)c1cn(Br)c2ccccc12)c1cccc(Cl)c1</smiles>

3f, $55 \%$<smiles>O=C(/C(=N/c1ccccc1)c1cn(Br)c2ccccc12)c1ccc(Br)cc1</smiles>

3j, $65 \%$<smiles>Cc1cccc(N=C(C(=O)c2ccccc2)c2cn(Br)c3ccccc23)c1</smiles>

3n, $72 \%$<smiles>O=C(/C(=N/c1ccccc1)c1cn(Br)c2cc(Cl)ccc12)c1ccccc1</smiles>

$3 r, 66 \%$<smiles></smiles>

3c, $69 \%$<smiles>O=C(/C(=N/c1ccccc1)c1cn(Br)c2ccccc12)c1ccc(Cl)cc1</smiles>

$3 g, 68 \%$<smiles>O=C(/C(=N/c1ccccc1)c1cn(Br)c2ccccc12)c1ccc(I)cc1</smiles>

3k, $73 \%$<smiles>Cc1ccc(N=C(C(=O)c2ccccc2)c2cn(Br)c3ccccc23)cc1</smiles>

3o, $74 \%$

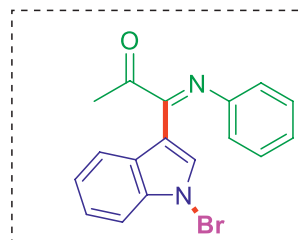

3s, $0 \%$<smiles>COc1ccc(C(=O)/C(=N/c2ccccc2)c2cn(Br)c3ccccc23)cc1</smiles>

3d, $74 \%$<smiles>O=C(/C(=N/c1ccccc1)c1cn(Br)c2ccccc12)c1ccccc1Br</smiles>

$3 h, 63 \%$<smiles>O=C(/C(=N/c1ccccc1)c1cn(Br)c2ccccc12)c1ccc(C(F)(F)F)cc1</smiles>

3I, $58 \%$<smiles>O=C(/C(=N/c1ccc(Cl)cc1)c1cn(Br)c2ccccc12)c1ccccc1</smiles>

$3 p, 59 \%$

${ }^{a}$ Reaction condition: All reactions were carried out with a-amino aryl ketones (1) (0.3 mmol), $1 \mathrm{H}$-indole (2a) $(0.3 \mathrm{mmol}), \mathrm{CBr} 4$ (0.6 mmol), and $\mathrm{NaOH}(1.2 \mathrm{mmol})$ in $\mathrm{MeCN}(2 \mathrm{ml})$ at room temperature for $12 \mathrm{~h}$.

${ }^{b}$ Isolated yield.

found that different electron-donating groups and electronwithdrawing groups have little effect on the reaction, and the yields of the reaction are all in the middle to good range. These results indicate that the electronic effect has no obvious influence on the yield. In addition, aryl-alkyl $\alpha$-amino aryl ketones, such as 1-(phenylamino)propan-2-one and 2-(isopropylamino)-1phenylethan-1-one, were selected as the starting material groups to be tested. Unfortunately, the reactions did not proceed smoothly, and these compounds were unable to obtain the corresponding target products.

In order to investigate the reaction mechanism, several control experiments were carried out (Scheme 2). 1) After adding 2,2,6,6tetramethyl piperidine nitroxide (TEMPO) to the model reaction, the yield of the target product 3a dropped from 68 to 5\%, and the reaction was basically completely inhibited. It means that this reaction may be a free radical reaction; 2) when 2-(methyl 


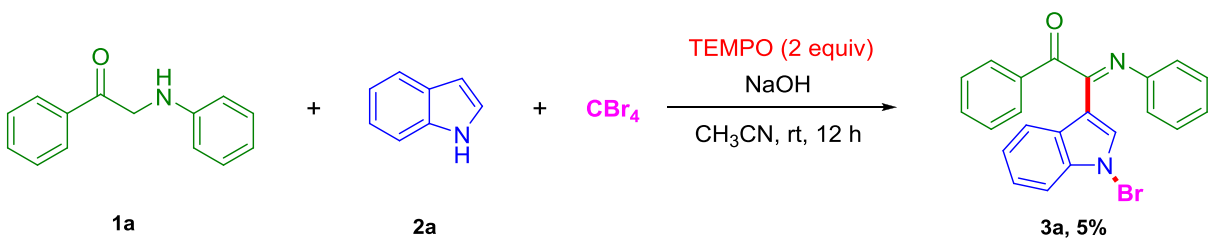<smiles>CN(c1ccccc1)C(C(=O)c1ccccc1)c1cn(Br)c2ccccc12</smiles>

1a

2a

N.R

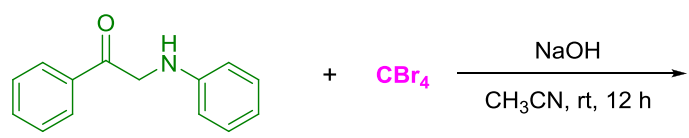

$1 \mathbf{a}$<smiles>CN(C)O[N+](C)(C)C(C)(C)C</smiles><smiles>O=C(/C=N/c1ccccc1)c1ccccc1</smiles>

Detected by GC-MS

SCHEME 2 | Control experiments.

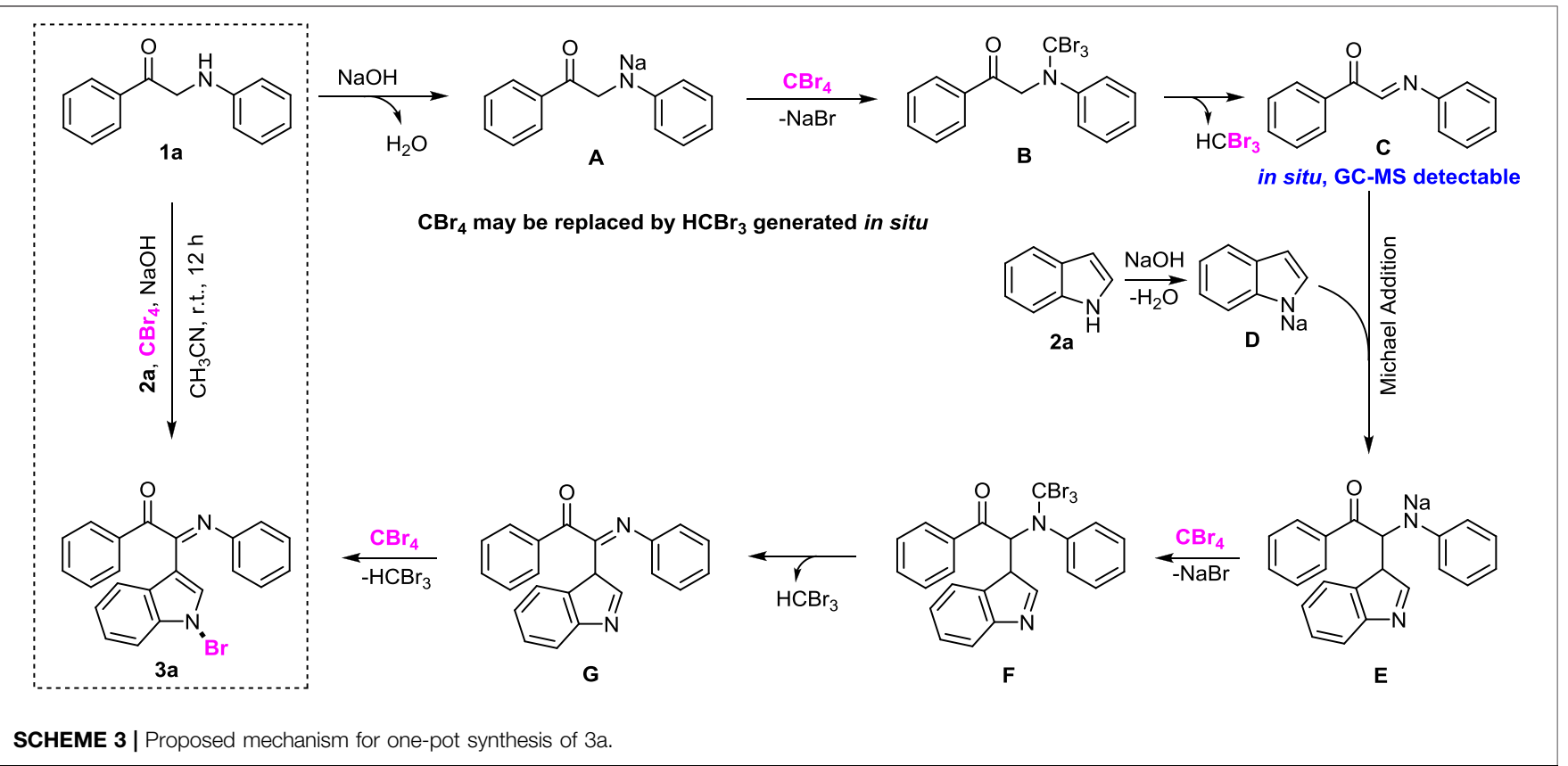

(phenyl)amino)-1-phenylethan-1-one is used instead of 1-phenyl2-(phenylamino)ethan-1-one (1a) under the standard conditions, the possible product (S)-2-(1-bromo- $1 H$-indol-3-yl)-2-(methyl (phenyl)amino)-1-phenylethan-1-one was not observed; 3) in addition, the 1-phenyl-2-(phenylamino)ethan-1-one (1a) reacted with $\mathrm{CBr}_{4}$ for $12 \mathrm{~h}$ under standard conditions, and the solution was monitored by GC-MS to detect (E)-1-phenyl-2-(phenylimino) ethane-1-one. This result implied that (E)-1-phenyl-2(phenylimino)ethane-1-ketone possibly is also an intermediate of three-component reaction.
Based on the aforementioned control experiments and literature reports (Liu et al., 2018; Zhou et al., 2017), a plausible mechanism is proposed for the one-pot synthesis of 2-(1-bromo-1H-indol-3-yl)-2-imino-carbonyls (Scheme 3). Initially, 1-phenyl-2-(phenylamino)ethan-1-one (1a) undergoes nucleophilic substitution reaction under the action of $\mathrm{NaOH}$ as a base to obtain intermediates $\mathrm{A}$. Then intermediate $\mathrm{A}$ forms intermediate $\mathrm{B}$ in the presence of $\mathrm{CBr}_{4}$, and intermediate $\mathrm{B}$ undergoes reduction and elimination to form imine intermediate C (Liu et al., 2016). At the same time, 
intermediate $C$ reacts with intermediate $D$ from indole (2a) in the presence of sodium hydroxide to give the Michael addition product $\mathrm{E}$. Then intermediate $\mathrm{E}$ forms intermediate $\mathrm{F}$ in the presence of $\mathrm{CBr}_{4}$, and intermediate $\mathrm{F}$ undergoes reduction and elimination to form imine intermediate G. Finally, G can easily afford $3 \mathrm{a}$ as a final product involving oxidation of $\mathrm{CBr}_{4}$. It should be noted that $\mathrm{HCBr}_{3}$ easily reacts with two equivalents of $\mathrm{NaOH}$ to form $\mathrm{HC}(\mathrm{O}) \mathrm{Br}$, which can be captured by aniline as $\mathrm{PhNHC}(\mathrm{O}) \mathrm{H}$ ( $\mathrm{N}$-formanilide) and is clearly detected by GC-MS (see SI). In addition, since only two equivalents of $\mathrm{CBr}_{4}$ were added in the reaction system, we proposed that $\mathrm{HCBr}_{3}$ could be working as $\mathrm{CBr}_{4}$ to react with $\mathrm{A}$ or $\mathrm{E}$ in some cases.

\section{CONCLUSION}

In summary, a mild C-H functionalization for the one-pot threecomponent synthesis of 2-(1-bromo- $1 H$-indol-3-yl)-2-iminocarbonyls is described. The reaction provides an efficient and practical method for the synthesis of biologically significant 2-(1-bromo-1H-indol-3-yl)-2-imino-carbonyls in an atom-economic manner under mild and simple reaction conditions. We are currently focusing on applying this $\mathrm{C}-\mathrm{H}$ bond functionalization to other inert bond cleavage reactions and further exploring research in the construction of more variable compounds.

\subsection{Experimental Section}

\subsubsection{General Information}

All commercially available reagents were used without further purification. Nuclear magnetic resonance (NMR) spectra were acquired at $298 \mathrm{~K}$ on ${ }^{1} \mathrm{H}$ NMR $(400 \mathrm{MHz})$ and ${ }^{13} \mathrm{C} \mathrm{NMR}$ $(101 \mathrm{MHz})$ Bruker NMR spectrometer with the sample dissolved in DMSO- $d_{6}$. All values of chemical shift were reported in parts per million ( $\mathrm{ppm}$ ) relative to the solvent signal with the coupling constant $(J)$ reported in Hertz. All compounds were characterized by ${ }^{1} \mathrm{H} \mathrm{NMR},{ }^{13} \mathrm{C} \mathrm{NMR}$, and EI or HRMS (double focusing mass analyzer). Column chromatography was performed on silica gel (300-400 mesh) using petroleum ether (PE)/ethyl acetate (EA) as a developing solvent.

\subsubsection{Synthesis of 2-(1-bromo-1H-indol-3-yl)-2-imino- carbonyls 3}

The mixture of $\alpha$-amino aryl ketones ( $0.3 \mathrm{mmol}), 1 \mathrm{H}$-indole (2a) $(0.3 \mathrm{mmol}), \mathrm{CBr}_{4}(0.6 \mathrm{mmol})$, and $\mathrm{NaOH}(1.2 \mathrm{mmol})$ in $\mathrm{MeCN}$ $(2 \mathrm{ml})$ was stirred at room temperature for $12 \mathrm{~h}$. The reaction was monitored by TLC. After the completion, the resulting mixture was separated with EA. Water was added for washing, and then $15 \mathrm{ml}$ of EA was used three times for extraction and liquid separation. The collected organic phase was dried with anhydrous $\mathrm{Na}_{2} \mathrm{SO}_{4}$, filtered, and the organic phase was distilled off under reduced pressure. The obtained products 3 were separated by a silica gel column layer, and mobile phase using petroleum ether with the fraction at $60-90^{\circ} \mathrm{C}$, and three purified products were obtained. The characterization data of all the products are given as follows.

\subsubsection{1 (E)-2-(1-bromo-1H-indol-3-yl)-1-phenyl-2- (phenylimino)ethan-1-one (3a)}

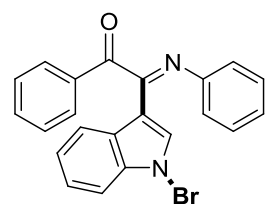

Yellow oil. Yield $62 \mathrm{mg}(68 \%)$ at $0.3 \mathrm{mmol}$ scale. ${ }^{1} \mathrm{H}$ NMR $\left(400 \mathrm{MHz}, \mathrm{DMSO}-d_{6}\right) \delta 8.41(\mathrm{~d}, J=7.8 \mathrm{~Hz}, 1 \mathrm{H}), 7.81(\mathrm{~d}, J=7.5$ $\mathrm{Hz}, 2 \mathrm{H}), 7.71(\mathrm{~s}, 1 \mathrm{H}), 7.64(\mathrm{t}, J=7.2 \mathrm{~Hz}, 1 \mathrm{H}), 7.55(\mathrm{~d}, J=7.1 \mathrm{~Hz}$, $1 \mathrm{H}), 7.43(\mathrm{dq}, J=15.1,7.6 \mathrm{~Hz}, 4 \mathrm{H}), 7.14(\mathrm{t}, J=7.2 \mathrm{~Hz}, 2 \mathrm{H}), 6.93(\mathrm{t}$, $J=7.1 \mathrm{~Hz}, 1 \mathrm{H}), 6.85(\mathrm{~d}, J=7.5 \mathrm{~Hz}, 2 \mathrm{H}) .{ }^{13} \mathrm{C} \mathrm{NMR}(101 \mathrm{MHz}$, DMSO- $\left.d_{6}\right) \delta 190.35,150.42,146.08,136.22,134.54,133.64$, $130.05,129.93,129.35,129.31,126.29,125.58,124.93,124.67$, $121.35,119.73,116.49$, 98.36. HRMS-ESI $(\mathrm{m} / \mathrm{z})$ : calcd for $\mathrm{C}_{22} \mathrm{H}_{15} \mathrm{BrN}_{2} \mathrm{O}[\mathrm{M}+\mathrm{H}]^{+}:$403.0441; found, 403.0444 .

\subsubsection{2 (E)-2-(1-bromo-1H-indol-3-yl)-2-(phenylimino)-1- (o-tolyl)ethan-1-one (3b)}

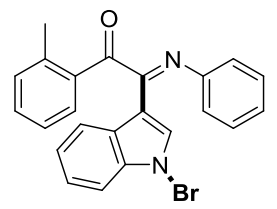

Yellow oil. Yield $87 \mathrm{mg}(86 \%)$ at $0.3 \mathrm{mmol}$ scale. ${ }^{1} \mathrm{H} \mathrm{NMR}$ $\left(400 \mathrm{MHz}, \mathrm{DMSO}-d_{6}\right) \delta 8.42(\mathrm{~d}, J=7.8 \mathrm{~Hz}, 1 \mathrm{H}), 7.78(\mathrm{~s}, 1 \mathrm{H}), 7.64$ $(\mathrm{d}, J=7.2 \mathrm{~Hz}, 1 \mathrm{H}), 7.54(\mathrm{~d}, J=6.2 \mathrm{~Hz}, 2 \mathrm{H}), 7.41(\mathrm{q}, J=6.9,6.3 \mathrm{~Hz}$, $3 \mathrm{H}), 7.27-7.16(\mathrm{~m}, 4 \mathrm{H}), 7.11(\mathrm{t}, J=7.7 \mathrm{~Hz}, 2 \mathrm{H}), 6.90(\mathrm{t}, J=7.6 \mathrm{~Hz}$, $1 \mathrm{H}), 6.76(\mathrm{~d}, J=7.7 \mathrm{~Hz}, 2 \mathrm{H}), 2.37(\mathrm{~s}, 3 \mathrm{H}) .{ }^{13} \mathrm{C} \mathrm{NMR}(101 \mathrm{MHz}$, DMSO- $\left.d_{6}\right) \delta 191.94,151.49,146.16,140.96,134.83,134.68,132.84$, $132.71,129.38,129.14,127.06,126.17,125.85,124.73,124.56$, $121.15,119.68,116.53,98.25,21.40$. HRMS-ESI (m/z): calcd for $\mathrm{C}_{23} \mathrm{H}_{17} \mathrm{BrN}_{2} \mathrm{O}[\mathrm{M}+\mathrm{H}]^{+}$: 417.0597; found, 417.0587.

3.1.2.3 (E)-2-(1-bromo-1H-indol-3-yl)-2-(phenylimino)-1(p-tolyl)ethan-1-one (3c)

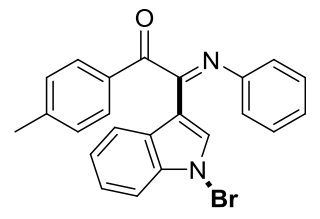

Yellow oil. Yield $70 \mathrm{mg}(69 \%)$ at $0.3 \mathrm{mmol}$ scale. ${ }^{1} \mathrm{H}$ NMR $\left(400 \mathrm{MHz}, \mathrm{DMSO}-d_{6}\right) \delta 8.42(\mathrm{~d}, J=7.9 \mathrm{~Hz}, 1 \mathrm{H}), 7.73$ (d, $J=7.8$ $\mathrm{Hz}, 2 \mathrm{H}), 7.67$ (s, 1H), 7.55 (d, $J=7.3 \mathrm{~Hz}, 1 \mathrm{H}), 7.42$ (p, $J=7.1$ $\mathrm{Hz}, 2 \mathrm{H}), 7.28$ (d, J=7.8 Hz, 2H), 7.17 (t, J= 7.5 Hz, 2H), 6.95 $(\mathrm{t}, J=7.3 \mathrm{~Hz}, 1 \mathrm{H}), 6.88(\mathrm{~d}, J=7.7 \mathrm{~Hz}, 2 \mathrm{H}), 2.30(\mathrm{~s}, 3 \mathrm{H}) \cdot{ }^{13} \mathrm{C}$ NMR $\left(101 \mathrm{MHz}, \mathrm{DMSO}-d_{6}\right) \delta 189.73,150.56,147.39,146.16$, $134.52,131.27,130.56,130.21,129.33,129.31,126.25,125.51$, $124.89,124.61,121.35,119.72,116.47,98.30,21.87$. HRMS- 
ESI $(\mathrm{m} / \mathrm{z})$ : calcd for $\mathrm{C}_{23} \mathrm{H}_{17} \mathrm{BrN}_{2} \mathrm{O}[\mathrm{M}+\mathrm{H}]^{+}$: 417.0597; found, 417.0603 .

3.1.2.4 (E)-2-(1-bromo-1H-indol-3-yl)-1-(4-methoxyphenyl)2-(phenylimino)ethan-1-one (3d)

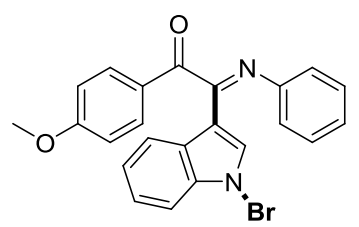

Yellow oil. Yield $78 \mathrm{mg}(74 \%)$ at $0.3 \mathrm{mmol}$ scale. ${ }^{1} \mathrm{H}$ NMR $\left(400 \mathrm{MHz}, \mathrm{DMSO}-d_{6}\right) \delta 8.41(\mathrm{~d}, J=8.1 \mathrm{~Hz}, 1 \mathrm{H}), 7.79$ $(\mathrm{d}, J=8.8 \mathrm{~Hz}, 2 \mathrm{H}), 7.65(\mathrm{~s}, 1 \mathrm{H}), 7.53(\mathrm{~d}, J=8.2 \mathrm{~Hz}, 1 \mathrm{H}), 7.45-$ $7.35(\mathrm{~m}, 2 \mathrm{H}), 7.16(\mathrm{t}, J=7.8 \mathrm{~Hz}, 2 \mathrm{H}), 6.95(\mathrm{dd}, J=13.3,8.1 \mathrm{~Hz}$, $3 \mathrm{H}), 6.87(\mathrm{~d}, J=7.5 \mathrm{~Hz}, 2 \mathrm{H}), 3.76(\mathrm{~s}, 3 \mathrm{H}) .{ }^{13} \mathrm{C} \mathrm{NMR}(101 \mathrm{MHz}$, DMSO- $\left.d_{6}\right) \delta 187.75,165.04,150.26,145.79,134.02,132.27$, $128.83,128.79,126.15,125.71,125.02,124.34,124.04,120.83$, 119.19, 115.97, 114.85, 97.69, 55.79. HRMS-ESI (m/z): calcd for $\mathrm{C}_{23} \mathrm{H}_{17} \mathrm{BrN}_{2} \mathrm{O}_{2}[\mathrm{M}+\mathrm{H}]^{+}$: 433.0546; found, 433.0562 .

3.1.2.5 (E)-2-(1-bromo-1H-indol-3-yl)-1-(4-fluorophenyl)-2(phenylimino)ethan-1-one (3e)

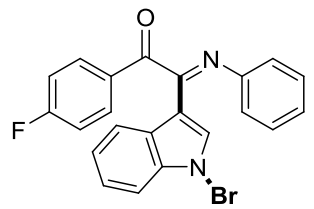

Yellow oil. Yield $73 \mathrm{mg}(58 \%)$ at $0.3 \mathrm{mmol}$ scale. ${ }^{1} \mathrm{H}$ NMR $\left(400 \mathrm{MHz}, \mathrm{DMSO}-d_{6}\right) \delta 8.46(\mathrm{~d}, J=7.6 \mathrm{~Hz}, 1 \mathrm{H}), 7.96(\mathrm{~d}, J=6.7$ $\mathrm{Hz}, 2 \mathrm{H}), 7.78(\mathrm{~s}, 1 \mathrm{H}), 7.59(\mathrm{~d}, J=7.3 \mathrm{~Hz}, 1 \mathrm{H}), 7.47(\mathrm{q}, J=8.3 \mathrm{~Hz}$, $2 \mathrm{H}), 7.33(\mathrm{t}, J=8.6 \mathrm{~Hz}, 2 \mathrm{H}), 7.20(\mathrm{t}, J=7.7 \mathrm{~Hz}, 2 \mathrm{H}), 6.98(\mathrm{t}, J=$ $7.3 \mathrm{~Hz}, 1 \mathrm{H}), 6.88(\mathrm{~d}, J=7.3 \mathrm{~Hz}, 2 \mathrm{H}) .{ }^{13} \mathrm{C} \mathrm{NMR}(101 \mathrm{MHz}$, DMSO- $\left.d_{6}\right) \delta 188.77,167.96,150.26,146.08,134.57,133.47(\mathrm{~d}$, $J=10.3 \mathrm{~Hz}), 130.57(\mathrm{~d}, J=2.5 \mathrm{~Hz}), 129.35,126.30,125.72$, 124.97, 124.71, 121.30, 119.71, 117.33, 117.11, 116.58, 98.39. HRMS-ESI (m/z): calcd for $\mathrm{C}_{22} \mathrm{H}_{14} \mathrm{BrFN}_{2} \mathrm{O}[\mathrm{M}+\mathrm{H}]^{+}$: 421.0346; found, 421.0337 .

3.1.2.6 (E)-2-(1-bromo-1H-indol-3-yl)-1-(3-chlorophenyl)-2(phenylimino)ethan-1-one (3f)

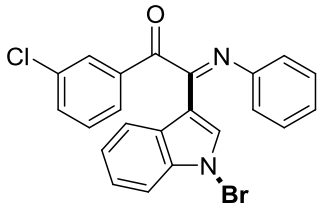

Yellow oil. Yield $72 \mathrm{mg}(55 \%)$ at $0.3 \mathrm{mmol}$ scale. ${ }^{1} \mathrm{H}$ NMR $\left(400 \mathrm{MHz}, \mathrm{DMSO}-d_{6}\right) \delta 8.48(\mathrm{~d}, J=7.9 \mathrm{~Hz}, 1 \mathrm{H}), 7.85-7.78(\mathrm{~m}$, $3 \mathrm{H}), 7.74(\mathrm{~d}, J=8.1 \mathrm{~Hz}, 1 \mathrm{H}), 7.59(\mathrm{~d}, J=7.7 \mathrm{~Hz}, 1 \mathrm{H}), 7.49(\mathrm{tt}, J=$ 15.1, 7.3 Hz, 3H), $7.20(\mathrm{t}, J=7.3 \mathrm{~Hz}, 2 \mathrm{H}), 6.99(\mathrm{t}, J=7.5 \mathrm{~Hz}, 1 \mathrm{H})$, $6.88(\mathrm{~d}, J=7.8 \mathrm{~Hz}, 2 \mathrm{H}) .{ }^{13} \mathrm{C}$ NMR $\left(101 \mathrm{MHz}, \mathrm{DMSO}-d_{6}\right) \delta$ $189.35,149.82,145.98,135.82,135.36,134.64,134.61,131.92$, $129.68,129.43,129.31,128.90,126.32,125.89,125.10,124.76$,
121.34, 119.70, 116.68, 98.44. HRMS-ESI (m/z): calcd for $\mathrm{C}_{22} \mathrm{H}_{14} \mathrm{BrClN}_{2} \mathrm{O}[\mathrm{M}+\mathrm{H}]^{+}$: 437.0051; found, 437.0052 .

\subsubsection{7 (E)-2-(1-bromo-1H-indol-3-yl)-1-(4-chlorophenyl)- 2-(phenylimino)ethan-1-one (3g)}<smiles>O=C(/C(=N/c1ccccc1)c1cn(Br)c2ccccc12)c1ccc(Cl)cc1</smiles>

Yellow oil. Yield $73 \mathrm{mg}(68 \%)$ at $0.3 \mathrm{mmol}$ scale. ${ }^{1} \mathrm{H}$ NMR $\left(400 \mathrm{MHz}, \mathrm{DMSO}-d_{6}\right) \delta 8.44(\mathrm{~d}, J=8.0 \mathrm{~Hz}, 1 \mathrm{H}), 7.82(\mathrm{~d}, J=8.4$ $\mathrm{Hz}, 2 \mathrm{H}), 7.75$ (s, 1H), 7.52 (dd, $J=12.0,7.9 \mathrm{~Hz}, 3 \mathrm{H}), 7.42$ (p, $J=$ $7.1 \mathrm{~Hz}, 2 \mathrm{H}), 7.15(\mathrm{t}, J=7.7 \mathrm{~Hz}, 2 \mathrm{H}), 6.94(\mathrm{t}, J=7.4 \mathrm{~Hz}, 1 \mathrm{H}), 6.84$ $(\mathrm{d}, J=7.8 \mathrm{~Hz}, 2 \mathrm{H}) .{ }^{13} \mathrm{C}$ NMR $\left(101 \mathrm{MHz}, \mathrm{DMSO}-d_{6}\right) \delta 189.29$, $150.10,146.00,141.18,134.59,132.40,131.90,130.09,129.39$, $129.37,126.29,125.68,125.02,124.71,121.33,119.70,116.59$, 98.47. HRMS-ESI $(\mathrm{m} / \mathrm{z})$ : calcd for $\mathrm{C}_{22} \mathrm{H}_{14} \mathrm{BrClN}_{2} \mathrm{O}_{2}[\mathrm{M}+\mathrm{H}]^{+}$: 437.0051; found, 437.0059.

3.1.2.8 (E)-2-(1-bromo-1H-indol-3-yl)-1-(2-bromophenyl)-2(phenylimino)ethan-1-one $(3 \mathrm{~h})$

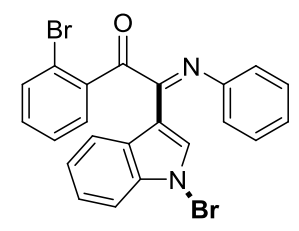

Yellow oil. Yield $91 \mathrm{mg}(63 \%)$ at $0.3 \mathrm{mmol}$ scale. ${ }^{1} \mathrm{H}$ NMR $\left(400 \mathrm{MHz}, \mathrm{DMSO}-d_{6}\right) \delta 8.47(\mathrm{~d}, J=7.9 \mathrm{~Hz}, 1 \mathrm{H}), 7.86(\mathrm{~d}, J=7.7$ $\mathrm{Hz}, 2 \mathrm{H}), 7.75(\mathrm{~d}, J=2.0 \mathrm{~Hz}, 1 \mathrm{H}), 7.68(\mathrm{t}, J=7.5 \mathrm{~Hz}, 1 \mathrm{H}), 7.59$ $(\mathrm{d}, J=7.5 \mathrm{~Hz}, 1 \mathrm{H}), 7.47(\mathrm{dq}, J=15.0,7.4 \mathrm{~Hz}, 4 \mathrm{H}), 7.19(\mathrm{t}, J=7.7$ $\mathrm{Hz}, 2 \mathrm{H}), 6.97(\mathrm{t}, J=7.5 \mathrm{~Hz}, 1 \mathrm{H}), 6.90(\mathrm{~d}, J=7.6 \mathrm{~Hz}, 2 \mathrm{H}) .{ }^{13} \mathrm{C}$ NMR $\left(101 \mathrm{MHz}, \mathrm{DMSO}-d_{6}\right) \delta 190.37,150.45,146.11,136.22$, $134.57,133.66,130.08,129.93,129.38,129.32,126.29,125.61$, $124.93,124.67,121.37,119.74,116.54,98.39$. HRMS-ESI (m/ $\mathrm{z}$ ): calcd for $\mathrm{C}_{22} \mathrm{H}_{14} \mathrm{Br}_{2} \mathrm{~N}_{2} \mathrm{O}[\mathrm{M}+\mathrm{H}]^{+}$: 480.9546; found, 480.9568 .

\subsubsection{9 (E)-2-(1-bromo-1H-indol-3-yl)-1-(3-bromophenyl)-} 2-(phenylimino)ethan-1-one (3i)

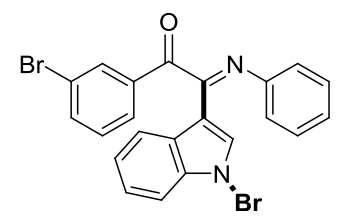

Yellow oil. Yield $94 \mathrm{mg}(65 \%)$ at $0.3 \mathrm{mmol}$ scale. ${ }^{1} \mathrm{H}$ NMR $\left(400 \mathrm{MHz}, \mathrm{DMSO}-d_{6}\right) \delta 7.69(\mathrm{~d}, J=8.0 \mathrm{~Hz}, 1 \mathrm{H}), 7.16(\mathrm{~s}, 1 \mathrm{H}), 7.09$ - $7.00(\mathrm{~m}, 3 \mathrm{H}), 6.79(\mathrm{~d}, J=7.5 \mathrm{~Hz}, 1 \mathrm{H}), 6.66(\mathrm{q}, J=7.8 \mathrm{~Hz}, 3 \mathrm{H})$, $6.42(\mathrm{t}, J=7.7 \mathrm{~Hz}, 2 \mathrm{H}), 6.20(\mathrm{t}, J=7.5 \mathrm{~Hz}, 1 \mathrm{H}), 6.09(\mathrm{~d}, J=7.4 \mathrm{~Hz}$, $3 \mathrm{H}) .{ }^{13} \mathrm{C}$ NMR $\left(101 \mathrm{MHz}, \mathrm{DMSO}-d_{6}\right) \delta 189.27,149.77,145.99$, $138.67,135.48,134.65,132.08,131.80,129.64,129.43,126.30$, $125.89,125.11,124.75,122.97,121.34,119.70,116.69,98.45$. 
HRMS-ESI (m/z): calcd for $\mathrm{C}_{22} \mathrm{H}_{14} \mathrm{Br}_{2} \mathrm{~N}_{2} \mathrm{O}[\mathrm{M}+\mathrm{H}]^{+}$: 480.9546 ; found, 480.9549 .

3.1.2.10 (E)-2-(1-bromo-1H-indol-3-yl)-1-(4-bromophenyl)2-(phenylimino)ethan-1-one (3j)<smiles>O=C(/C(=N/c1ccccc1)c1cn(Br)c2ccccc12)c1ccc(Br)cc1</smiles>

Yellow oil. Yield $78 \mathrm{mg}(65 \%)$ at $0.3 \mathrm{mmol}$ scale. ${ }^{1} \mathrm{H}$ NMR $\left(400 \mathrm{MHz}, \mathrm{DMSO}-d_{6}\right) \delta 8.44(\mathrm{~d}, J=8.0 \mathrm{~Hz}, 1 \mathrm{H}), 7.79-7.70(\mathrm{~m}$, $3 \mathrm{H}), 7.67$ (d, $J=8.5 \mathrm{~Hz}, 2 \mathrm{H}), 7.55(\mathrm{~d}, J=7.3 \mathrm{~Hz}, 1 \mathrm{H}), 7.43$ (p, $J=$ $7.1 \mathrm{~Hz}, 2 \mathrm{H}), 7.16(\mathrm{t}, J=7.7 \mathrm{~Hz}, 2 \mathrm{H}), 6.95(\mathrm{t}, J=7.4 \mathrm{~Hz}, 1 \mathrm{H}), 6.84$ $(\mathrm{d}, J=7.9 \mathrm{~Hz}, 2 \mathrm{H}) .{ }^{13} \mathrm{C}$ NMR $\left(101 \mathrm{MHz}, \mathrm{DMSO}-d_{6}\right) \delta 189.54$, $150.08,145.99,134.58,133.05,132.70,131.88,130.69,129.38$, $126.30,125.70,125.03,124.72,121.33,119.71,116.59,98.47$. HRMS-ESI (m/z): calcd for $\mathrm{C}_{22} \mathrm{H}_{14} \mathrm{Br}_{2} \mathrm{~N}_{2} \mathrm{O}[\mathrm{M}+\mathrm{H}]^{+}$: 480.9546; found, 480.9565 .

3.1.2.11 (E)-2-(1-bromo-1H-indol-3-yl)-1-(4-iodophenyl)-2(phenylimino)ethan-1-one (3k)<smiles>O=C(/C(=N/c1ccccc1)c1cn(Br)c2ccccc12)c1ccc(I)cc1</smiles>

Yellow solid. Yield $98 \mathrm{mg}(73 \%)$ at $0.3 \mathrm{mmol}$ scale. ${ }^{1} \mathrm{H}$ NMR $\left(400 \mathrm{MHz}, \mathrm{DMSO}-d_{6}\right) \delta 8.44(\mathrm{~d}, J=7.8 \mathrm{~Hz}, 1 \mathrm{H}), 7.87(\mathrm{~d}, J=7.7 \mathrm{~Hz}$, 2H), $7.74(\mathrm{~s}, 1 \mathrm{H}), 7.57(\mathrm{~d}, J=7.7 \mathrm{~Hz}, 3 \mathrm{H}), 7.44(\mathrm{p}, J=7.0 \mathrm{~Hz}, 2 \mathrm{H})$, $7.18(\mathrm{t}, J=7.3 \mathrm{~Hz}, 2 \mathrm{H}), 6.97(\mathrm{t}, J=7.2 \mathrm{~Hz}, 1 \mathrm{H}), 6.86(\mathrm{~d}, J=7.6 \mathrm{~Hz}$, $2 \mathrm{H}) .{ }^{13} \mathrm{C}$ NMR $\left(101 \mathrm{MHz}, \mathrm{DMSO}-d_{6}\right) \delta 189.93,150.11,145.99$, $138.92,134.56,132.92,131.37,129.38,126.30,125.65,125.02$, $124.72,121.34,119.72,116.57,106.15,98.47$. HRMS-ESI $(\mathrm{m} / \mathrm{z})$ : calcd for $\mathrm{C}_{22} \mathrm{H}_{14} \mathrm{BrIN}_{2} \mathrm{O}[\mathrm{M}+\mathrm{H}]^{+}$: 528.9407; found, 528.9415 .

\subsubsection{2 (E)-2-(1-bromo-1H-indol-3-yl)-2-(phenylimino)} -1-(4-(trifluoromethyl)phenyl)ethan-1-one (3l)<smiles>O=C(/C(=N/c1ccccc1)c1cn(Br)c2ccccc12)c1ccc(C(F)(F)F)cc1</smiles>

Yellow solid. Yield $82 \mathrm{mg}(58 \%)$ at $0.3 \mathrm{mmol}$ scale. ${ }^{1} \mathrm{H}$ NMR $\left(400 \mathrm{MHz}, \mathrm{DMSO}-d_{6}\right) \delta 8.52(\mathrm{~d}, J=7.9 \mathrm{~Hz}, 1 \mathrm{H}), 8.06(\mathrm{~d}, J=8.1$ $\mathrm{Hz}, 2 \mathrm{H}), 7.87(\mathrm{~d}, J=7.1 \mathrm{~Hz}, 3 \mathrm{H}), 7.61(\mathrm{~d}, J=7.4 \mathrm{~Hz}, 1 \mathrm{H}), 7.50$ (p, $J=7.2 \mathrm{~Hz}, 2 \mathrm{H}), 7.20(\mathrm{t}, J=7.8 \mathrm{~Hz}, 2 \mathrm{H}), 6.99(\mathrm{t}, J=7.5 \mathrm{~Hz}, 1 \mathrm{H})$, $6.89(\mathrm{~d}, J=7.6 \mathrm{~Hz}, 2 \mathrm{H}) .{ }^{13} \mathrm{C}$ NMR $\left(101 \mathrm{MHz}, \mathrm{DMSO}-d_{6}\right) \delta$ $189.84,149.91,145.85,136.68,130.93,129.41,126.86,126.73$, $126.37,125.85,125.14(\mathrm{~d}, J=4.1 \mathrm{~Hz}), 124.83,121.37,119.73$, 116.68, 98.57. HRMS-ESI (m/z): calcd for $\mathrm{C}_{23} \mathrm{H}_{14} \mathrm{BrF}_{3} \mathrm{~N}_{2} \mathrm{O}[\mathrm{M}+$ $\mathrm{H}]^{+}$: 471.0314; found, 471.0322.
3.1.2.13 (E)-2-(1-bromo-1H-indol-3-yl)-1-(naphthalen-2-yl)2-(phenylimino)ethan-1-one (3m)

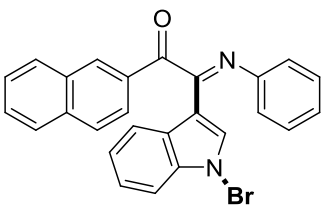

Yellow oil. Yield $52 \mathrm{mg}(47 \%)$ at $0.3 \mathrm{mmol}$ scale. ${ }^{1} \mathrm{H}$ NMR $\left(400 \mathrm{MHz}, \mathrm{DMSO}-d_{6}\right) \delta 8.56(\mathrm{~s}, 1 \mathrm{H}), 8.46(\mathrm{~d}, J=7.6 \mathrm{~Hz}, 1 \mathrm{H}), 8.14$ $(\mathrm{d}, J=8.1 \mathrm{~Hz}, 1 \mathrm{H}), 7.94(\mathrm{t}, J=8.0 \mathrm{~Hz}, 2 \mathrm{H}), 7.83(\mathrm{~d}, J=8.6 \mathrm{~Hz}, 1 \mathrm{H})$, $7.78(\mathrm{~s}, 1 \mathrm{H}), 7.67(\mathrm{t}, J=7.2 \mathrm{~Hz}, 1 \mathrm{H}), 7.57(\mathrm{t}, J=7.8 \mathrm{~Hz}, 2 \mathrm{H}), 7.44$ $(\mathrm{q}, J=7.8,7.4 \mathrm{~Hz}, 2 \mathrm{H}), 7.09(\mathrm{t}, J=7.3 \mathrm{~Hz}, 2 \mathrm{H}), 6.91(\mathrm{~d}, J=7.5 \mathrm{~Hz}$, $2 \mathrm{H}), 6.86(\mathrm{t}, J=7.1 \mathrm{~Hz}, 1 \mathrm{H}) .{ }^{13} \mathrm{C}$ NMR $\left(101 \mathrm{MHz}, \mathrm{DMSO}-d_{6}\right) \delta$ $190.38,150.51,146.35,136.45,134.71,134.16,132.42,131.11$, $130.65,130.61,129.71,129.38,129.29,128.26,127.89,126.23$, $125.79,124.82,124.62,123.54,121.27,119.71,116.59,98.29$. HRMS-ESI (m/z): calcd for $\mathrm{C}_{26} \mathrm{H}_{17} \mathrm{BrN}_{2} \mathrm{O}[\mathrm{M}+\mathrm{H}]^{+}$: 453.0597; found, 453.0604 .

\subsubsection{4 (E)-2-(1-bromo-1H-indol-3-yl)-1-phenyl-2-} (m-tolylimino)ethan-1-one (3n)<smiles>Cc1cccc(/N=C(/C(=O)c2ccccc2)c2cn(Br)c3ccccc23)c1</smiles>

Yellow oil. Yield $90 \mathrm{mg}(72 \%)$ at $0.3 \mathrm{mmol}$ scale. ${ }^{1} \mathrm{H}$ NMR $\left(400 \mathrm{MHz}, \mathrm{DMSO}-d_{6}\right) \delta 8.48(\mathrm{~d}, J=8.0 \mathrm{~Hz}, 1 \mathrm{H}), 7.86(\mathrm{~d}, J=7.7$ $\mathrm{Hz}, 2 \mathrm{H}), 7.75$ (s, 1H), 7.69 (t, $J=7.5 \mathrm{~Hz}, 1 \mathrm{H}), 7.59$ (d, $J=7.4 \mathrm{~Hz}$, $1 \mathrm{H}), 7.48(\mathrm{dt}, J=27.2,7.5 \mathrm{~Hz}, 4 \mathrm{H}), 7.06(\mathrm{t}, J=7.8 \mathrm{~Hz}, 1 \mathrm{H}), 6.79(\mathrm{~d}$, $J=7.6 \mathrm{~Hz}, 1 \mathrm{H}), 6.74(\mathrm{~s}, 1 \mathrm{H}), 6.68(\mathrm{~d}, J=7.7 \mathrm{~Hz}, 1 \mathrm{H}), 2.16(\mathrm{~s}, 3 \mathrm{H})$. ${ }^{13} \mathrm{C}$ NMR $\left(101 \mathrm{MHz}, \mathrm{DMSO}-d_{6}\right) \delta 190.41,150.27,146.03,138.64$, $136.18,134.56,133.74,130.05,129.91,129.35,129.13,126.26$, $125.64,125.59,124.64,122.09,119.72,118.35,116.52,98.29$, 21.28. HRMS-ESI $(\mathrm{m} / \mathrm{z})$ : calcd for $\mathrm{C}_{23} \mathrm{H}_{17} \mathrm{BrN}_{2} \mathrm{O}[\mathrm{M}+\mathrm{H}]^{+}$: 417.0597; found, 417.0588.

\subsubsection{5 (E)-2-(1-bromo-1H-indol-3-yl)-1-phenyl-2- (p-tolylimino)ethan-1-one (3o)}

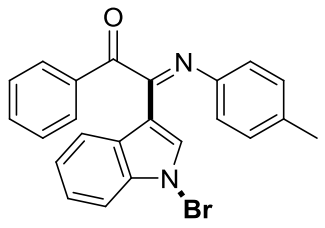

Yellow oil. Yield $92 \mathrm{mg}(74 \%)$ at $0.3 \mathrm{mmol}$ scale. ${ }^{1} \mathrm{H}$ NMR (400 MHz, DMSO- $\left.d_{6}\right) \delta 8.47(\mathrm{~d}, J=8.0 \mathrm{~Hz}, 1 \mathrm{H}), 7.87$ (d, $J=7.3$ $\mathrm{Hz}, 2 \mathrm{H}), 7.73(\mathrm{~s}, 1 \mathrm{H}), 7.69$ (t, $J=7.5 \mathrm{~Hz}, 1 \mathrm{H}), 7.59$ (d, $J=7.3 \mathrm{~Hz}$, $1 \mathrm{H}), 7.51(\mathrm{t}, J=7.3 \mathrm{~Hz}, 2 \mathrm{H}), 7.46(\mathrm{t}, J=8.3 \mathrm{~Hz}, 2 \mathrm{H}), 7.00(\mathrm{~d}, J=7.5$ $\mathrm{Hz}, 2 \mathrm{H}), 6.82(\mathrm{~d}, J=7.7 \mathrm{~Hz}, 2 \mathrm{H}), 2.16(\mathrm{~s}, 3 \mathrm{H}) .{ }^{13} \mathrm{C} \mathrm{NMR}$ $\left(101 \mathrm{MHz}, \mathrm{DMSO}-d_{6}\right) \delta 190.68,150.23,143.50,136.24,134.54$, $134.02,133.60,130.06,129.98,129.84,129.33,126.24,125.54$, $124.59,121.32,119.71,116.49,98.22,79.66,20.80$. HRMS-ESI 
(m/z): calcd for $\mathrm{C}_{23} \mathrm{H}_{17} \mathrm{BrN}_{2} \mathrm{O}[\mathrm{M}+\mathrm{H}]^{+}$: 417.0597; found, 417.0588 .

3.1.2.16 (E)-2-(1-bromo-1H-indol-3-yl)-2-((4-chlorophenyl) imino)-1-phenylethan-1-one (3p)

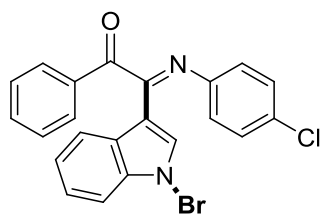

Yellow oil. Yield $77 \mathrm{mg}(59 \%)$ at $0.3 \mathrm{mmol}$ scale. ${ }^{1} \mathrm{H}$ NMR $\left(400 \mathrm{MHz}, \mathrm{DMSO}-d_{6}\right) \delta 8.48(\mathrm{~d}, J=8.2 \mathrm{~Hz}, 1 \mathrm{H}), 7.87(\mathrm{~d}, J=7.7$ $\mathrm{Hz}, 2 \mathrm{H}), 7.72(\mathrm{~d}, J=7.6 \mathrm{~Hz}, 2 \mathrm{H}), 7.54(\mathrm{t}, J=7.6 \mathrm{~Hz}, 2 \mathrm{H}), 7.43-$ $7.32(\mathrm{~m}, 3 \mathrm{H}), 7.26(\mathrm{~d}, J=8.9 \mathrm{~Hz}, 2 \mathrm{H}), 6.92(\mathrm{~d}, J=8.0 \mathrm{~Hz}, 2 \mathrm{H}), 6.81$ $(\mathrm{d}, J=3.4 \mathrm{~Hz}, 1 \mathrm{H}) .{ }^{13} \mathrm{C}$ NMR $\left(101 \mathrm{MHz}\right.$, DMSO- $\left.d_{6}\right) \delta 190.50$, $151.77,145.51,136.37,135.07,133.67,130.85,130.12,129.96$, $129.25,128.81,126.64,125.00,124.07,123.27,121.76,116.48$, 109.32. HRMS-ESI (m/z): calcd for $\mathrm{C}_{22} \mathrm{H}_{14} \mathrm{BrClN}_{2} \mathrm{O}[\mathrm{M}+\mathrm{H}]^{+}$: 437.0051; found, 437.0040.

3.1.2.17 (E)-2-(1-bromo-5-methyl-1H-indol-3-yl)-1-phenyl-2(phenylimino)ethan-1-one (3q)<smiles>Cc1ccc2c(c1)c(/C(=N\c1ccccc1)C(=O)c1ccccc1)cn2Br</smiles>

Yellow solid. M.P. $158-159^{\circ} \mathrm{C}$. Yield $97 \mathrm{mg}(78 \%)$ at $0.3 \mathrm{mmol}$ scale. ${ }^{1} \mathrm{H}$ NMR (400 MHz, DMSO- $\left.d_{6}\right) \delta 8.33(\mathrm{~d}, J=8.6 \mathrm{~Hz}, 1 \mathrm{H}), 7.84$ $(\mathrm{d}, J=7.7 \mathrm{~Hz}, 2 \mathrm{H}), 7.68(\mathrm{t}, J=6.0 \mathrm{~Hz}, 2 \mathrm{H}), 7.50(\mathrm{t}, J=7.7 \mathrm{~Hz}, 2 \mathrm{H})$, $7.37(\mathrm{~s}, 1 \mathrm{H}), 7.29(\mathrm{~d}, J=8.5 \mathrm{~Hz}, 1 \mathrm{H}), 7.18(\mathrm{t}, J=7.7 \mathrm{~Hz}, 2 \mathrm{H}), 6.96(\mathrm{t}$, $J=7.5 \mathrm{~Hz}, 1 \mathrm{H}), 6.88(\mathrm{~d}, J=7.7 \mathrm{~Hz}, 2 \mathrm{H}), 2.47(\mathrm{~s}, 3 \mathrm{H}) .{ }^{13} \mathrm{C} \mathrm{NMR}$ $\left(101 \mathrm{MHz}, \mathrm{DMSO}-d_{6}\right) \delta 190.43,150.37,146.19,136.20,134.08$, $133.68,132.83,130.04,129.94,129.55,129.31,127.57,125.55$, $124.86,121.40,119.40,116.26,98.15,21.41$. HRMS-ESI $(\mathrm{m} / \mathrm{z})$ : calcd for $\mathrm{C}_{23} \mathrm{H}_{17} \mathrm{BrN}_{2} \mathrm{O}[\mathrm{M}+\mathrm{H}]^{+}$: 417.0597; found, 417.0593.

3.1.2.18 (E)-2-(1-bromo-6-chloro-1H-indol-3-yl)-1-phenyl-2(phenylimino)ethan-1-one (3r)

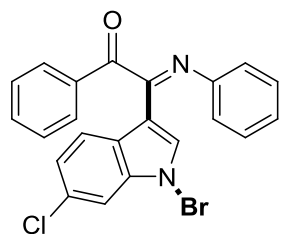

Yellow oil. Yield $86 \mathrm{mg}(66 \%)$ at $0.3 \mathrm{mmol}$ scale. ${ }^{1} \mathrm{H}$ NMR $\left(400 \mathrm{MHz}, \mathrm{DMSO}-d_{6}\right) \delta 8.65(\mathrm{t}, J=2.0 \mathrm{~Hz}, 1 \mathrm{H}), 7.88(\mathrm{~d}, J=6.8$ $\mathrm{Hz}, 2 \mathrm{H}), 7.78(\mathrm{~d}, J=1.9 \mathrm{~Hz}, 1 \mathrm{H}), 7.70(\mathrm{t}, J=7.5 \mathrm{~Hz}, 1 \mathrm{H}), 7.62(\mathrm{dd}$, $J=8.5,1.9 \mathrm{~Hz}, 1 \mathrm{H}), 7.55-7.49(\mathrm{~m}, 3 \mathrm{H}), 7.21(\mathrm{t}, J=7.8 \mathrm{~Hz}, 2 \mathrm{H})$, $7.00(\mathrm{t}, J=7.5 \mathrm{~Hz}, 1 \mathrm{H}), 6.92(\mathrm{~d}, J=7.6 \mathrm{~Hz}, 2 \mathrm{H}) .{ }^{13} \mathrm{C}$ NMR $\left(101 \mathrm{MHz}, \mathrm{DMSO}-d_{6}\right) \delta 189.91,150.52,145.86,136.31,134.75$, $133.53,130.86,130.20,129.92,129.38,128.29,126.76,125.14$,
125.06, 121.34, 121.15, 116.61, 97.94. HRMS-ESI (m/z): calcd for $\mathrm{C}_{22} \mathrm{H}_{14} \mathrm{BrClN}_{2} \mathrm{O}[\mathrm{M}+\mathrm{H}]^{+}$: 437.0051; found, 437.0036.

\subsubsection{Synthesis of 2-Bromoacetophenones}

The mixture of acetophenone $(1.2 \mathrm{~g}, 10 \mathrm{mmol}), \mathrm{N}$-bromosuccinimide (NBS) $(1.958 \mathrm{~g}, 11 \mathrm{mmol})$, and $p$-toluenesulfonic acid (TsOH) $(0.172 \mathrm{~g}$, $1 \mathrm{mmol})$ in $\mathrm{MeCN}(120 \mathrm{ml})$ was heated at $60^{\circ} \mathrm{C}$ for $24 \mathrm{~h}$. The reaction was monitored by TLC. After the reaction was completed, the solvent was distilled off under reduced pressure, then $30 \mathrm{ml}$ of saturated $\mathrm{NaHCO}_{3}$ aqueous solution was poured into the residue, and the mixture was extracted with ethyl acetate $(E A)(3 \times 20 \mathrm{ml})$. Next, the organic phases were combined, and anhydrous $\mathrm{Na}_{2} \mathrm{SO}_{4}$ was added for drying. Finally, a rotary evaporator was used to distill the organic solvent under reduced pressure, leaving its residue without further treatment and purification, and it was saved for the next step. Without additional instructions, other substituted acetophenones are similar to this synthesis method.

\subsubsection{Synthesis of a-amino Aryl Ketones (1a-1 h)}

Under nitrogen atmosphere, the mixture of 2-bromoacetophenones (199 mg, $10 \mathrm{mmol}$ ), aniline ( $84 \mathrm{mg}, 10 \mathrm{mmol}$ ), $\mathrm{NaHCO}_{3}(93 \mathrm{mg}$, $1 \mathrm{mmol})$, and $\mathrm{EtOH}(40 \mathrm{ml})$ was added into a dry round-bottom flask and heated at $25^{\circ} \mathrm{C}$ for $12 \mathrm{~h}$. The reaction was monitored by TLC. After the reaction was completed, the reaction mixture was filtered with suction, and the filtered solid was left in the upper layer, which was washed with $\mathrm{EtOH}(3 \times 5 \mathrm{ml})$. Finally, it was dissolved in ethyl acetate (EA) and distilled under reduced pressure to obtain an organic phase yellow solid.

\section{DATA AVAILABILITY STATEMENT}

The original contributions presented in the study are included in the article/Supplementary Material; further inquiries can be directed to the corresponding authors.

\section{AUTHOR CONTRIBUTIONS}

JX and WD conceived of the presented idea. DC and YL collected the data. DC and HL wrote the first draft of the manuscript. YL prepared the figures. JX, RQ, and $\mathrm{WD}$ contributed to manuscript revision.

\section{FUNDING}

We are grateful for the financial support from the National Natural Science Foundation of China (Nos. 21502049 and 51573040), the Hunan Provincial Natural Science Foundation of China (No. 2018JJ2032), and the Planned Science and Technology Project of Hunan Province, China (No. 2015WK3003).

\section{SUPPLEMENTARY MATERIAL}

The Supplementary Material for this article can be found online at: https://www.frontiersin.org/articles/10.3389/fchem.2022.825772/ full\#supplementary-material 


\section{REFERENCES}

Bandini, M., and Eichholzer, A. (2009). Catalytic Functionalization of Indoles in a New Dimension. Angew. Chem. Int. Edition 48, 9608-9644. doi:10.1002/anie. 200901843

Bartoli, G., Bencivenni, G., and Dalpozzo, R. (2010). Organocatalytic Strategies for the Asymmetric Functionalization of Indoles. Chem. Soc. Rev. 39, 4449-4465. doi:10.1039/B923063G

Bell, R., Carmeli, S., and Sar, N. (1994). Vibrindole A, a Metabolite of the Marine Bacterium, Vibrio Parahaemolyticus, Isolated from the Toxic Mucus of the Boxfish Ostracion Cubicus. J. Nat. Prod. 57, 1587-1590. doi:10.1021/np50113a022

Contractor, R., Samudio, I. J., Estrov, Z., Harris, D., McCubrey, J. A., Safe, S. H., et al. (2005). A Novel Ring-Substituted Diindolylmethane,1,1-Bis[3'-(5Methoxyindolyl)]-1-(p-t-Butylphenyl) Methane, Inhibits Extracellular SignalRegulated Kinase Activation and Induces Apoptosis in Acute Myelogenous Leukemia. Cancer Res. 65, 2890-2898. doi:10.1158/0008-5472.can-04-3781

Dalpozzo, R. (2015). Strategies for the Asymmetric Functionalization of Indoles: an Update. Chem. Soc. Rev. 44, 742-778. doi:10.1039/C4CS00209A

Deka, B., Deb, M. L., and Baruah, P. K. (2020). Recent Advances on the C2Functionalization of Indole via Umpolung. Top. Curr. Chem. (Z) 378, 22. doi:10.1007/s41061-020-0287-7

Girard, S. A., Knauber, T., and Li, C.-J. (2014). The Cross-Dehydrogenative Coupling of C Sp 3H Bonds: A Versatile Strategy for CC Bond Formations. Angew. Chem. Int. Ed. 53, 74-100. doi:10.1002/anie.201304268

Joucla, L., and Djakovitch, L. (2009). Transition Metal-Catalysed, Direct and Site-Selective N1-, C2- or C3-Arylation of the Indole Nucleus: 20 Years of Improvements. Adv. Synth. Catal. 351, 673-714. doi:10.1002/ adsc. 200900059

Kumar, P., Nagtilak, P. J., and Kapur, M. (2021). Transition Metal-Catalyzed C-H Functionalizations of Indoles. New J. Chem. 45, 13692-13746. doi:10.1039/ d1nj01696b

Leitch, J. A., Bhonoah, Y., and Frost, C. G. (2017). Beyond C2 and C3: TransitionMetal-Catalyzed C-H Functionalization of Indole. ACS Catal. 7, 5618-5627. doi:10.1021/acscatal.7b01785

Li, C.-J. (2009). Cross-Dehydrogenative Coupling (CDC): Exploring C-C Bond Formations beyond Functional Group Transformations. Acc. Chem. Res. 42, 335-344. doi:10.1021/ar800164n

Liu, X.-X., Wu, Z.-Y., He, Y.-Q., Zhou, X.-Q., Hu, T., Ma, C.-W., et al. (2016). Copper-Catalyzed C-N Bond Formation via Oxidative Cross-Coupling of Amines with a-Aminocarbonyl Compounds. Adv. Synth. Catal. 358, 2385-2391. doi:10.1002/adsc.201600132

Liu, X., Pu, J., Luo, X., Cui, X., Wu, Z., and Huang, G. (2018). CBr4-mediated Cross-Coupling Reactions of $\alpha$-amino Carbonyl Compounds with Alcohols and Thiols to Build C-O and C-S Bonds, Respectively. Org. Chem. Front. 5, 361-365. doi:10.1039/c7qo00830a

Ohfune, Y. (1992). Stereoselective Routes toward the Synthesis of Unusual Amino Acids. Acc. Chem. Res. 25, 360-366. doi:10.1021/ar00020a006

Ou, Y., Yang, T., Tang, N., Yin, S.-F., Kambe, N., and Qiu, R. (2021). Photo-Induced N-N Coupling of O-Nitrobenzyl Alcohols and Indolines to Give N-Aryl-1-Amino Indoles. Org. Lett. 23, 6417-6422. doi:10.1021/acs.orglett.1c02227

Paira, P., Hazra, A., Kumar, S., Paira, R., Sahu, K. B., Naskar, S., et al. (2009). Efficient Synthesis of 3,3-diheteroaromatic Oxindole Analogues and Their in vitro Evaluation for Spermicidal Potential. Bioorg. Med. Chem. Lett. 19, 4786-4789. doi:10.1016/j.bmcl.2009.06.049

Phipps, R. J., Grimster, N. P., and Gaunt, M. J. (2008). Cu(II)-Catalyzed Direct and Site-Selective Arylation of Indoles Under Mild Conditions. J. Am. Chem. Soc. 130, 8172-8174. doi:10.1021/ja801767s

Sandtorv, A. H. (2015). Transition Metal-Catalyzed CH Activation of Indoles. Adv. Synth. Catal. 357, 2403-2435. doi:10.1002/adsc.201500374

Sashidhara, K. V., Kumar, M., Sonkar, R., Singh, B. S., Khanna, A. K., and Bhatia, G. (2012). Indole-Based Fibrates as Potential Hypolipidemic and Antiobesity Agents. J. Med. Chem. 55, 2769-2779. doi:10.1021/jm201697v
Scheuermann, C. J. (2010). Beyond Traditional Cross Couplings: The Scope of the Cross Dehydrogenative Coupling Reaction. Chem. Asian J. 5, 436-451. doi:10. 1002/asia.200900487

Sivaprasad, G., Perumal, P. T., Prabavathy, V. R., and Mathivanan, N. (2006). Synthesis and Anti-microbial Activity of Pyrazolylbisindoles-Promising Antifungal Compounds. Bioorg. Med. Chem. Lett. 16, 6302-6305. doi:10.1016/j. bmcl.2006.09.019

Song, S., Zhang, Y., Yeerlan, A., Zhu, B., Liu, J., and Jiao, N. (2017). Cs2 CO3 -Catalyzed Aerobic Oxidative Cross-Dehydrogenative Coupling of Thiols with Phosphonates and Arenes. Angew. Chem. Int. Ed. 56, 2487-2491. doi:10.1002/ anie. 201612190

Tang, Z., Liu, Z., Tong, Z., Xu, Z., Au, C.-T., Qiu, R., et al. (2019). Cu-Catalyzed Cross-Dehydrogenative Coupling of Heteroaryl C(sp2)-H and Tertiary C(sp3)$\mathrm{H}$ Bonds for the Construction of All-Carbon Triaryl Quaternary Centers. Org. Lett. 21, 5152-5156. doi:10.1021/acs.orglett.9b01755

Van Order, R. B., and Lindwall, H. G. (1942). Indole. Chem. Rev. 30, 69-96. doi:10. $1021 /$ cr60095a004

Wang, F., Zhou, Q., Zhang, X., and Fan, X. (2021). Direct $\alpha$-Alkenylation of Cyclic Amines with Maleimides through Fe(III)-Catalyzed C(sp3)-H/C(sp2)-H Cross Dehydrogenative Coupling. J. Org. Chem. 86, 11708-11722. doi:10.1021/acs.joc. $1 \mathrm{c} 01198$

Wang, Z.-Q., Hu, M., Huang, X.-C., Gong, L.-B., Xie, Y.-X., and Li, J.-H. (2012). Direct $a$-Arylation of a-Amino Carbonyl Compounds with Indoles Using Visible Light Photoredox Catalysis. J. Org. Chem. 77, 8705-8711. doi:10. 1021/jo301691h

Wu, J.-C., Song, R.-J., Wang, Z.-Q., Huang, X.-C., Xie, Y.-X., and Li, J.-H. (2012). Copper-Catalyzed $\mathrm{CH}$ Oxidation/Cross-Coupling of a-Amino Carbonyl Compounds. Angew. Chem. Int. Ed. 51, 3453-3457. doi:10.1002/anie.201109027

Yang, T., Lu, H., Shu, Y., Ou, Y., Hong, L., Au, C.-T., et al. (2020). CF3SO2NaMediated, UV-Light-Induced Friedel-Crafts Alkylation of Indoles with Ketones/Aldehydes and Bioactivities of Products. Org. Lett. 22, 827-831. doi:10.1021/acs.orglett.9b04272

Ye, Y., Kevlishvili, I., Feng, S., Liu, P., and Buchwald, S. L. (2020). Highly Enantioselective Synthesis of Indazoles with a C3-Quaternary Chiral Center Using CuH Catalysis. J. Am. Chem. Soc. 142, 10550-10556. doi:10.1021/jacs.0c04286

Yeung, C. S., and Dong, V. M. (2011). Catalytic Dehydrogenative Cross-Coupling: Forming Carbon-Carbon Bonds by Oxidizing Two Carbon-Hydrogen Bonds. Chem. Rev. 111, 1215-1292. doi:10.1021/cr100280d

Zhang, Y., Ni, M. J., and Feng, B. N., (2016). Iron-Catalyzed Direct $\alpha$-Arylation of a-Amino Carbonyl Compounds With Indoles. Org. Biomol. Chem. 14, 1550-1554. doi:10.1039/C5OB02325D

Zhou, X., Ma, H., Shi, C., Zhang, Y., Liu, X., and Huang, G. (2017). Facile Synthesis of Polysubstituted Imidazoles through CBr4-Mediated Tandem Cyclization of Amidines with 1,3-Dicarbonyl Compounds or Ketones. Eur. J. Org. Chem. 2017, 237-240. doi:10.1002/ejoc.201601428

Conflict of Interest: The authors declare that the research was conducted in the absence of any commercial or financial relationships that could be construed as a potential conflict of interest.

Publisher's Note: All claims expressed in this article are solely those of the authors and do not necessarily represent those of their affiliated organizations, or those of the publisher, the editors, and the reviewers. Any product that may be evaluated in this article, or claim that may be made by its manufacturer, is not guaranteed or endorsed by the publisher.

Copyright (C) 2022 Chen, Lu, Liu, Deng, Qiu and Xiang. This is an open-access article distributed under the terms of the Creative Commons Attribution License (CC BY). The use, distribution or reproduction in other forums is permitted, provided the original author(s) and the copyright owner(s) are credited and that the original publication in this journal is cited, in accordance with accepted academic practice. No use, distribution or reproduction is permitted which does not comply with these terms. 